\title{
HIGHLIGHTS
}

MEDICAL ONCOLOGY

\section{Carcinoembryonic antigen mRNA expression-a marker for early pancreatic cancer recurrence?}

Improved means of identifying patients with pancreatic adenocarcinoma who do not benefit from resection would protect these individuals from unnecessary pancreatectomy. Kelly et al., from the Memorial Sloan-Kettering Cancer Center, now report that reverse transcription (RT)-PCR-based detection of peritoneal micrometastases identifies a subgroup of patients with pancreatic cancer who might benefit from nonsurgical treatment as opposed to resection. "Conventional cytology is currently accepted for assessment of peritoneal micrometastatic disease," explain the investigators, "however, cytology lacks sensitivity, because many patients with negative cytology develop early peritoneal recurrence."

The researchers, led by principal investigator Daniel Coit, analyzed carcinoembryonic antigen (CEA) mRNA expression-a marker of pancreatic cancer-by RT-PCR from peritoneal washings of 115 patients with pancreatic cancer undergoing diagnostic laparoscopy. The investigators identified 42 individuals with micrometastatic disease based on CEA positivity in comparison to 22 patients identified by cytology or biopsy of gross disease. In addition, 11 of 13 patients with peritoneal disease identified by standard staging laproscopy were CEA positive, while 31 individuals were CEA positive with no other evidence of peritoneal metastases. Furthermore, in 62 patients who underwent $\mathrm{R} 0$ resection, 11 individuals identified as CEA positive by RT-PCR experienced a significantly higher rate of recurrence postoperatively than patients with negative CEA results at a median follow-up period of 10 months.

The researchers noted that indicators of advanced disease (including advanced clinical tumor stage, positive cytology and presence of peritoneal and hepatic metastases) and early disease recurrence both significantly correlated with CEA positivity. "If these observations can be expanded and validated, RT-PCR of

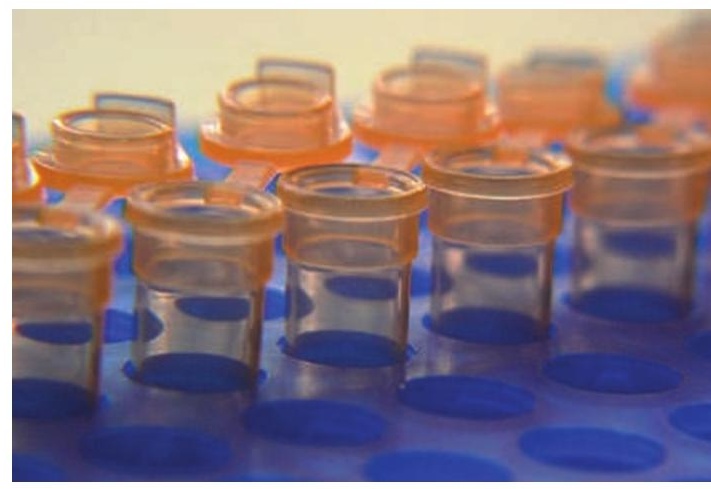

peritoneal washings may ... help select cancer patients at risk for early recurrence, for whom treatment other then upfront surgical resection may be appropriate" conclude the investigators.

Rowan Higgs

Original article Kelly, K. J. et al. Prognostic impact of RT-PCR-based detection of peritoneal micrometastases in patients with pancreatic cancer undergoing curative resection. Ann. Surg. Oncol. 16, 3333-3339 (2009) 